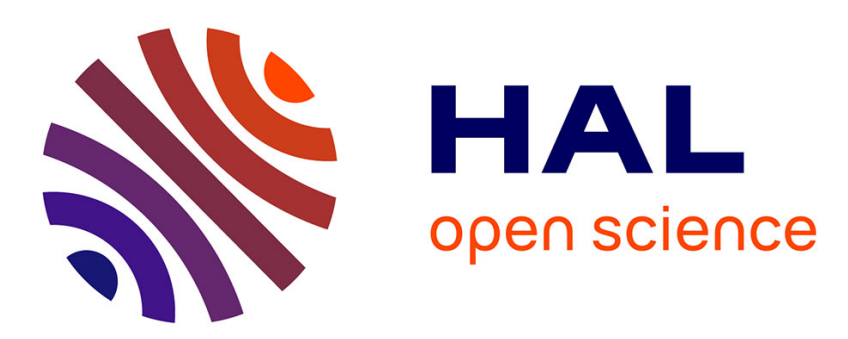

\title{
Financement et territorialisation des établissements de santé français: une relation dissymétrique
}

\author{
Laurent Mériade
}

\section{To cite this version:}

Laurent Mériade. Financement et territorialisation des établissements de santé français: une relation dissymétrique. Revue d'économie financière, 2019, 132, p. 197 à 214. 10.3917/ecofi.132.0197 . halshs02007377

\section{HAL Id: halshs-02007377 \\ https://shs.hal.science/halshs-02007377}

Submitted on 9 Dec 2019

HAL is a multi-disciplinary open access archive for the deposit and dissemination of scientific research documents, whether they are published or not. The documents may come from teaching and research institutions in France or abroad, or from public or private research centers.
L'archive ouverte pluridisciplinaire HAL, est destinée au dépôt et à la diffusion de documents scientifiques de niveau recherche, publiés ou non, émanant des établissements d'enseignement et de recherche français ou étrangers, des laboratoires publics ou privés. 


\title{
Financement et territorialisation des établissements de santé français : une relation dissymétrique
}

\begin{abstract}
Résumé
En France, au cours des trente dernières années, la question des relations entre les établissements de santé et leurs territoires est devenue essentielle dans la plupart des réformes de santé initiées. Par ailleurs, les modalités de financement de ces établissements semblent occuper, aujourd'hui, une place tellement centrale dans l'organisation sanitaire qu'il nous parait important de s'interroger sur leurs effets sur la territorialisation de ces établissements. Il s'agit du questionnement principal de ce travail que nous entreprenons à partir d'une lecture croisée du contenu des réformes du financement de ces établissements avec une étude longitudinale des principales statistiques nationales portant sur leur situation financière et leur offre de soins dans les territoires.
\end{abstract}

Mots clés : santé, financement, territorialisation, hôpital, territoire

\section{Financing and territorialisation of French health care institutions: an asymmetrical relationship}

\begin{abstract}
In France, over the past thirty years, the question of the relationship between health care institutions and their territories has become essential in most of the health reforms initiated. Moreover, the financing modalities of these institutions seem to occupy such a central place in the health organization that it seems important to us to question their effects on the territorialization of these institutions. This is the main questioning of this work that we are undertaking based on a cross-reading of the content of the reforms of the financing of these institutions with a longitudinal study of the main national statistics on their financial situation and care provision in the territories.
\end{abstract}

Keywords: health, financing, territorialization, hospital, territory

Code JEL : I18 R53 


\section{Introduction}

En France, depuis le début du XIXème siècle, la question de la territorialisation des politiques de santé demeure très marquée par le rôle de l'Etat et ces choix en matière de déconcentration ou de décentralisation. Depuis, maintenant une vingtaine d'années, il n'est quasiment pas de texte de loi ou d'orientation en santé qui ne fasse référence à une nécessaire " territorialisation» (loi HPST ${ }^{1}$, création des ARH - $\mathrm{ARS}^{2}, \mathrm{SROS}^{3}$ ). Dès lors, on parle de «territoires de soins », d' " hôpital de proximité", de "santé territoriale », etc. Le «territoire » apparait comme une référence obligée voire une incantation alors que parallèlement, en termes scientifiques comme pratiques, il soulève de nombreuses interrogations (Mériade et al, 2017).

En France, force est de constater que, malgré la volonté de développer des territoires de santé cohérents (tels que les $\mathrm{GHT}^{4}$ ), les impulsions restent fortement centralisées. Les grandes orientations de la territorialisation de la santé continuent à être définies au niveau du Ministère de la Santé et des ARS. La réflexion sur la construction des périmètres de santé demeure faible au niveau des territoires alors que c'est à cette échelle que la proximité entre les patients et les établissements est la plus grande (Rican et Vaillant, 2009).

La territorialisation de la santé est définie comme son appréhension à partir de réalités spatiales et des processus qui y sont liés (Amat-Roze, 2011). De manière plus concrète, il s'agit d'une adaptation de l'offre de service de santé à un territoire en tenant compte des principaux déterminants de ce territoire et de ses usagers (Fleuret, 2003). Les principaux travaux retiennent quatre déterminants de cette territorialisation, principalement liés à la personne (biologiques, liés au style de vie) ou à son environnement (déterminants communautaires et socio-environnementaux) (Power, 2005).

Parallèlement, cette territorialisation interfère également avec des systèmes de financement dont les effets sur la construction de l'offre de soins territoriale sont rarement mesurés. Ceci est particulièrement vrai concernant le financement des établissements de santé ${ }^{5}$ qui, depuis une vingtaine d'années, a connu d'importantes réformes (Tarification à l'activité, Missions d'intérêt général et, maintenant, financement au parcours de soin). Ces modes de financement produisent-ils des effets sur la territorialisation de ces établissements de santé ? et si oui, lesquels?

Pour aborder cette problématique, nous proposons d'effectuer une lecture croisée du contenu des dernières réformes du financement des établissements de santé français avec une étude longitudinale des principales statistiques nationales portant sur la santé financière des établissements et leurs offres de soin dans les territoires (Drees ${ }^{6}, \operatorname{Irdes}^{7}$, Insee $^{8}$, Dgfip $^{9}$ ).

Pour cela, notre article est structuré de la manière suivante. Dans un premier temps, nous revenons sur les évolutions historiques du financement des établissements de santé publics afin de rendre compte de leurs proximités avec la problématique de la territorialisation de la santé. Dans un deuxième temps, notre analyse croisée et longitudinale de données nous permet de mesurer plusieurs effets directs ou indirects des changements intervenus dans les modalités de financement des établissements de santé sur leur territorialisation.

\section{Le financement des établissements de santé : un détour historique}

Aujourd'hui, le financement des établissements de santé français repose sur deux piliers essentiels : l'activité et la nature des missions menées dans ces établissements. Cette situation est née de transformations progressives de la place des établissements de santé dans le paysage médical français.

Jusqu'au début des années 1940, l'hôpital est resté le lieu d'accueil exclusif des malades pauvres. Construit sur le modèle des «Hôtel Dieu», il demeurait un lieu d'hospice voire d'asile souvent fondé par une congrégation religieuse (Chauveau, 2011). L'hôpital était 
d'abord une institution sociale avant d'être une institution sanitaire. Il fallait être indigent ou accidenté du travail pour être admis (ibid.).

A partir de la Révolution française (loi des 16 et 24 août 1790) puis sous la Deuxième République (loi de 1851), le financement de ces hôpitaux ou hospices avait été progressivement confié aux corps municipaux. Ceci renforça le lien financier entre les territoires et leurs établissements de santé. L'État n'intervenant que par exception en cas de carence des communes ou pour prendre en charge les menaces épidémiques.

Ce n'est que pendant la Seconde guerre mondiale (loi du 21 décembre 1941), que l'hôpital s'ouvre aux malades payants et accélère sa transformation en établissement de soins organisé en services, avec à sa tête, une direction générale. L'hôpital passe d'une logique de secours aux pauvres à celle d'assistance aux malades ce qui lui permet de dynamiser ses ressources à partir de son territoire d'implantation.

La création de la Sécurité Sociale (Ordonnance $n^{\circ} 45-2250$ du 4 octobre 1945) consacrera ces évolutions en permettant aux hôpitaux de répondre à une demande de soins prise en charge par l'Assurance Maladie mais dans un cadre financier national.

Il faut attendre la loi du 31 décembre 1970 pour voir apparaitre une première planification territoriale de la santé qui permettra d'articuler les établissements sanitaires et la politique de santé publique. Les Schémas régionaux d'organisation sanitaire (devenus « de soins » en 2009) font leur apparition avec la loi du 31 juillet 1991 (loi Evin) qui instaure une carte sanitaire nationale avec la région comme référence principale.

Puis, en 1996, Les ordonnances «Juppé » remplacent la logique de moyens à l'hôpital par une logique de résultat pilotée par des agences de régulation régionales (ARH puis ARS). Ce changement de logique de gestion et de financement va se concrétiser par l'ordonnance de simplification du 4 septembre 2004 qui instaure le financement des établissements de santé selon un système de tarification à l'activité (T2A) inspiré du système DRG (Diagnosis Related Groups) mis en place aux Etats Unis dans le programme Medicare au début des années 1980 (De Pouvourville, 1990). Il s'agit d'un virage majeur dans le financement des établissements de santé qui place l'établissement de santé au centre d'une double contrainte de développement de son activité et de service public territorial.

Pour répondre à ce double défi, l'Etat, par la loi HPST ${ }^{10}$ (2009) va chercher à organiser et coordonner les acteurs de la Santé notamment à travers les Contrats Locaux de Santé (CLS) (Haschar-Noé et Salaméro 2016). En 2016, la création de 135 Groupements hospitaliers de territoires (GHT) concernant 850 établissements constitue une nouvelle tentative de construire des offres de soins territoriales coordonnées.

Il apparait que ces différentes réformes et changements législatifs ont toujours entretenus des rapports plus ou moins étroits avec le territoire sans que pour autant, en matière de financement, ces territoires soient réellement impliqués. Le plan santé 2022 (présenté par le président Macron en septembre 2018) confirme cette tendance (Fleuret, 2015) malgré sa volonté de développer progressivement un financement au parcours territorialisé de soins ${ }^{11}$.

\section{Le financement des établissements dans les territoires}

Jusqu'au début des années 2000, les hôpitaux publics recevaient chaque année une enveloppe budgétaire appelée «dotation globale ». Cette dotation était reconduite sur une base historique, évoluant principalement selon un taux de croissance relativement constant.

Depuis 2004, la France a fait le choix d'un financement mixte conjuguant le paiement à l'acte (majoritaire) et le paiement forfaitaire. La tarification à l'activité (T2A) assoit les budgets de ces établissements directement sur leur activité, c'est-à-dire sur le nombre et les caractéristiques des séjours réalisés dans l'année. De manière schématique, le PMSI réalise une classification de séjours en groupes homogènes de malades (GHM) qui dépendent du diagnostic médical, des actes pratiqués et le cas échéant de certaines caractéristiques du 
patient. Un tarif est alors associé à chaque GHM sous la forme d'un GHS (Groupe Homogène de Séjours). Dans les établissements publics comme privés, le financement d'exploitation correspond principalement aux recettes liées à cette tarification prise en charge par l'Assurance Maladie et les contributions complémentaires des mutuelles ou des patients. Aux côtés de ces financements à l'activité, les dotations forfaitaires MIGAC ${ }^{12}$ couvrent les missions d'intérêt général (missions d'enseignement et recherche, SAMU, SMUR, etc.).

De plus, depuis 2015, de nouvelles modalités de financement pour les hôpitaux de proximité ont été définies (Drees, 2017). Un forfait «activités isolées »a été instauré pour financer les activités nécessaires mais non rentables. Il est calculé à partir d'un niveau d'activité insuffisant pour atteindre l'équilibre financier. Les hôpitaux dits « de proximité » bénéficient aussi, à compter du 1er janvier 2016, de deux dotations : une dotation forfaitaire et une dotation organisationnelle et populationnelle calibrée sur les caractéristiques du territoire (ibid.). Mais l'ensemble de ces mesures sont surtout destinés à compenser les faiblesses de la T2A au niveau territorial.

\section{La territorialisation des établissements de santé}

La problématique de la territorialisation de la santé renvoie aux questions de l'organisation de de l'offre de soins sur un territoire (Fleuret, 2003) et de ses déterminants socio-économiques et individuels.

De manière générale, le choix d'un établissement de santé par un patient s'effectue plutôt à partir de l'avis de son médecin habituel et du critère de proximité de l'offre (McGuirk et Porell 1984). Par contre, Victoor et al., (2012) montre que le choix de la proximité est variable selon l'âge et le niveau d'éducation du patient. Plus celui-ci est jeune et son niveau d'éducation important, moins le la proximité est un critère de choix important (ibid.).

Néanmoins le critère de proximité est souvent cité comme un des facteurs les plus importants, voire le plus signifiant, dans le choix du service de santé consulté (Comber et al., 2011 ; Lacoste et Spinosi, 2002 ; Pilkington, 2012). Cependant, ce choix est modéré par l'importance de la distance aux établissements de santé. Plus la distance à l'établissement est élevée plus les patients ont tendance à aller encore plus loin. Ceci est particulièrement vrai dans le cas de l'accès aux maternités (Pilkington, op.cit.) Cela signifie aussi que la proximité est perçue de manière différente selon la région de résidence (Zeitlin et al., 2011), par exemple, selon que l'on habite à Paris ou en Haute Corrèze. Les patients peuvent ainsi choisir de recourir à des soins près de leur lieu de travail ou des lieux d'achats, lorsque les pratiques de mobilités sont quotidiennes (Salze et al., 2011; Vallée, 2014). Ainsi de nombreuses études soulignent l'importance de déterminants sociaux et économiques dans les perceptions de la proximité d'un établissement par les patients (Wellstood et al., 2006 ; Comber et al., op.cit.).

Par ailleurs, certains travaux mettent en évidence les liens forts qui existent entre le choix de la proximité et les catégories sociales (Lacoste et Spinosi, 2002), ou le fait de ne pas disposer d'une complémentaire santé (ibid.). D'autres travaux évoquent l'importance de la réputation de l'établissement de santé ou sa spécialisation pour lequel le consentement à se déplacer augmente (Com-Ruelle et al., 1989 ; Moschetti, 2005). Pour cela, un travail très conséquent (Barlet et al.,2012) a permis d'établir l'indicateur d'accessibilité potentielle localisée (APL) ${ }^{13}$ qui mesure la densité du nombre d'établissements accessibles pour une population (100 000 habitants) compte tenu de ses principales caractéristiques socio-professionnelles.

Les analyses des pratiques spatiales de recours aux soins sont donc particulièrement riches et illustrées notamment grâce à la prise en compte des caractéristiques individuelles des patients et du contexte géographique en matière d'offre de soins. Par contre, moins de travaux caractérise l'influence de déterminants propres au système de santé, alors que des réformes importantes, depuis une vingtaine d'années en France, ont modifié la manière dont les 
établissements fonctionnent et exercent leurs activités dans un territoire. Un de ces changements majeurs demeure le mode de financement des établissements.

\section{Méthodologie d'étude}

Des changements majeurs dans le financement des établissements de santé sont intervenus en 2004 avec la T2A. Suivre et analyser les caractéristiques de l'offre de soin hospitaliers et cliniques depuis 2003 jusqu'à aujourd'hui, apparait être un bon moyen de juger de l'impact des réformes financières successives sur la territorialisation. Pour cela, nous reprenons et agrégeons les données annuelles produites par plusieurs sources statistiques nationales en matière de santé (Drees, Irdes, Insee, Dgfip). Ces organismes produisent des données brutes relatives aux principales évolutions des établissements de santé français en matière d'activités médicale, socio-médicale et économique. Dans un premier temps, notre approche est descriptive en vue d'établir un panorama de la situation des établissements au niveau national et dans leurs territoires, afin, dans un deuxième temps, d'essayer de définir quelques hypothèses relatives aux effets des modes de financement sur la territorialisation des établissements.

Notre approche est centrée sur l'analyse de ces informations statistiques portant sur l'état de établissements de santé au cours des 15 dernières années. Nos champs d'analyse portent plus particulièrement sur la situation financière des établissements de santé français, leurs offres de soins ainsi que leurs capacités d'accueils et leurs activités. Nous croisons ces éléments avec les nombreuses transformations des modalités de financement de ces établissements intervenues durant cette période. Nous réalisons un focus spécifique sur les services d'urgence de ces établissements qui présentent la particularité, d'une part, d'être une "porte d'entrée » médicale de plus en plus utilisée par les patients d'un territoire et, d'autre part, de disposer d'un mode de financement mixte (à l'activité et à la dotation) qui agrège les deux principales modalités actuelles de financement des établissements de santé français.

\section{Une situation financière qui affecte la territorialisation}

A partir de 2005, le déficit des établissements de santé a progressé de manière continue (tableau 1). Ceci est particulièrement vrai dans les Centre Hospitaliers Régionaux et de villes moyennes qui, en nombre et en volume budgétaire, représentent la part la plus importante des établissements (environ 1000 établissements). Au total, la moitié environ des établissements publics sont déficitaires en 2016 contre à peine 5\% en 2002 -2003. Ceci produit au moins deux conséquences en matière de territorialisation. Premièrement, désormais, les hôpitaux contractualisent avec les ARS des $\mathrm{CPOM}^{14}$ ou des EPRD ${ }^{15}$ pour lesquels l'activité devient la principale variable d'ajustement bien avant la localisation et la diversité de l'offre de soins. Deuxièmement, même si l'offre de soins territoriale est prise en compte dans les PPI ${ }^{16}$, les marges de manœuvre financières se réduisent compte tenu de la dégradation de la capacité d'autofinancement des établissements.

L'essentiel de ces déficits reste concentré sur un petit nombre d'établissements. La moitié de ce déficit cumulé est, en 2016, imputable à seulement 40 établissements (contre 25 établissements en 2015). Ceci dénote un nombre réduit, mais en augmentation, d'établissements en très grande difficulté financière dans lesquels les efforts immédiats portent avant tout sur le redressement de la situation.

Parallèlement à la dégradation des résultats, l'encours de la dette des hôpitaux publics augmente légèrement pour atteindre 29,9 milliards d'euros en 2016 (29,7 milliards d'euros en 2015). Le taux d'endettement représente $51,2 \%$ des ressources stables (capitaux propres + emprunts) contre 33,1\% en 2003 (tableau 2), ce qui, en général, constitue un seuil d'alerte. L'endettement devenant supérieur aux capitaux propres et présentant un niveau de risque très élevé. 
Subséquemment, l'effort d'investissement, ratio des dépenses d'investissement rapportées aux recettes, est de 5,9\% en 2016 et il a été divisé par deux depuis 2009 (tableau 3). En 2016, les investissements représentent seulement 4,1 milliards d'euros en 2016. Dès lors, même si les établissements de proximité sont globalement maintenus en nombre, faute d'activité et donc de financement, leurs services aux usagers peuvent se réduire sensiblement. La territorialisation des établissements de santé ne peut être parfois qu'apparente et partielle. Un patient peut avoir un centre hospitalier en face de chez lui mais il peut n'y trouver aucun service dont il a besoin. Il s'agit de la territorialisation vécue qui, à l'image de l'espace vécu (Lefebvre 1974), peut être très éloignée de la territorialisation conçue ou perçue. Un des déterminants de cette dissonance, pas toujours observée, provient des capacités financières du système de santé et indirectement des choix en matière de mode de financement.

Tableau 1 : Excédent ou déficit des hôpitaux publics depuis 2002 (Sources : DGFiP)

\begin{tabular}{|c|c|c|c|c|c|c|c|c|c|c|c|c|c|c|c|}
\hline & 2002 & 2003 & 2004 & 2005 & 2006 & 2007 & 2008 & 2009 & 2010 & 2011 & 2012 & 2013 & 2014 & 2015 & 2016 \\
\hline Ensemble HP & 0,9 & 0,5 & 1,0 & 0,3 & $-0,4$ & $-0,9$ & $-0,6$ & $-0,4$ & $-0,4$ & $-0,6$ & 0,2 & $-0,2$ & $-0,4$ & $-0,6$ & $-0,3$ \\
\hline CHR & & & & & & & & & & & & & & & \\
\hline AP-HP & 0,8 & $-0,3$ & 1,6 & $-1,1$ & 2,7 & $-0,2$ & 0,2 & $-1,3$ & $-1,7$ & $-1,2$ & $-0,3$ & $-0,1$ & $-0,2$ & 0,6 & 0,9 \\
\hline $\begin{array}{c}\text { Autres centres } \\
\text { hospitaliers } \\
\text { régionaux }\end{array}$ & 0,5 & $-0,1$ & 0,4 & $-0,2$ & $-1,1$ & $-2,2$ & $-2,4$ & $-1,7$ & $-0,9$ & $-1,1$ & 0,2 & $-0,3$ & $-0,7$ & $-1,3$ & $-0,5$ \\
\hline Autres $\mathrm{CH}$ & & & & & & & & & & & & & & & \\
\hline Grands $\mathrm{CH}$ & 0,4 & 0,2 & 0,4 & 0,6 & $-1,3$ & $-1,4$ & $-0,5$ & $-0,1$ & $-0,2$ & $-0,4$ & 0,1 & 0,0 & $-0,3$ & $-0,7$ & $-0,3$ \\
\hline Moyens $\mathrm{CH}$ & 0,7 & 0,6 & 0,8 & 0,6 & $-0,9$ & $-0,7$ & $-0,3$ & 0,0 & $-0,7$ & $-1,1$ & 0,0 & $-0,7$ & $-0,9$ & $-0,9$ & $-1,7$ \\
\hline Petits $\mathrm{CH}$ & 2,2 & 1,6 & 2,0 & 1,1 & 0,8 & 0,9 & 1,0 & 1,2 & 0,9 & 0,5 & 0,4 & 0,7 & 0,5 & $-0,3$ & 0,3 \\
\hline $\begin{array}{l}\text { CH ex-hôpitaux } \\
\text { locaux }\end{array}$ & 2,0 & 1,6 & 2,5 & 2,0 & 2,1 & 2,3 & 2,2 & 2,5 & 2,3 & 1,4 & 1,3 & 0,7 & 0,6 & 0,7 & 0,7 \\
\hline $\begin{array}{l}\text { CH spécialisés en } \\
\text { psychiatrie }\end{array}$ & 3,2 & 3,1 & 2,3 & 1,2 & 0,4 & 0,7 & 0,7 & 0,6 & 0,6 & 0,6 & 0,4 & $-0,1$ & 0,0 & 0,3 & 1,0 \\
\hline
\end{tabular}

Tableau 2. Évolution du taux d'endettement des hôpitaux publics depuis 2002 (Source : DGFiP)

\begin{tabular}{|c|c|c|c|c|c|c|c|c|c|c|c|c|c|c|}
\hline \multicolumn{11}{|c}{ En \% des ressources stables ${ }^{1}$} \\
\hline $\mathbf{2 0 0 2}$ & $\mathbf{2 0 0 3}$ & $\mathbf{2 0 0 4}$ & $\mathbf{2 0 0 5}$ & $\mathbf{2 0 0 6}$ & $\mathbf{2 0 0 7}$ & $\mathbf{2 0 0 8}$ & $\mathbf{2 0 0 9}$ & $\mathbf{2 0 1 0}$ & $\mathbf{2 0 1 1}$ & $\mathbf{2 0 1 2}$ & $\mathbf{2 0 1 3}$ & $\mathbf{2 0 1 4}$ & $\mathbf{2 0 1 5}$ & $\mathbf{2 0 1 6}$ \\
\hline 32,9 & 33,1 & 34,0 & 34,7 & 36,4 & 39,7 & 43,3 & 46,0 & 47,4 & 48,7 & 49,6 & 49,8 & 50,0 & 50,5 & 51,5 \\
\hline
\end{tabular}

Tableau 3. Évolution de l'effort d'investissement des hôpitaux publics de 2002 à 2016 (Source : DGFiP)

\begin{tabular}{|c|c|c|c|c|c|c|c|c|c|c|c|c|c|c|}
\hline \multicolumn{10}{|c|}{ En \% des recettes } \\
\hline $\mathbf{2 0 0 2}$ & $\mathbf{2 0 0 3}$ & $\mathbf{2 0 0 4}$ & $\mathbf{2 0 0 5}$ & $\mathbf{2 0 0 6}$ & $\mathbf{2 0 0 7}$ & $\mathbf{2 0 0 8}$ & $\mathbf{2 0 0 9}$ & $\mathbf{2 0 1 0}$ & $\mathbf{2 0 1 1}$ & $\mathbf{2 0 1 2}$ & $\mathbf{2 0 1 3}$ & $\mathbf{2 0 1 4}$ & $\mathbf{2 0 1 5}$ & $\mathbf{2 0 1 6}$ \\
\hline 7,2 & 7,9 & 9,0 & 9,3 & 9,8 & 9,9 & 10,6 & 10,9 & 10,2 & 9,4 & 8,8 & 7,6 & 7,1 & 6,6 & 5,9 \\
\hline
\end{tabular}

\section{Des modes de financements privilégiant certaines formes d'activités hospitalières}

Entre 2009 et 2016, le nombre d'établissements de santé a significativement augmenté (tableau 4). Ceci s'explique, d'une part, par un changement de comptabilisation des CHR intervenu en 2015 qui prend en compte l'ensemble des sites et plus les entités juridiques (par exemple l'AP HP à Paris est une seule entité juridique mais elle compte de 39 sites 
hospitaliers) et, d'autre part, par une augmentation effective des sites hospitaliers. Cette dernière se concentre néanmoins principalement dans les zones urbaines et dans les établissements dans lesquels l'«activité » augmente.

Tableau 4. Nombre d'entités et capacités d'hospitalisation des établissements de santé par catégorie d'établissement en 2008 et 2016 (Source : adapté de Rapport Drees 2018)

\begin{tabular}{|l|r|r|}
\hline & Entités & Entités \\
$\mathbf{2 0 0 9}$
\end{tabular}

Ces divergences territoriales sont repérables dans le nombre de lits d'hospitalisation à temps complet installés (tableau 5). De 2003 à 2016, tous établissements, toutes disciplines et tous secteurs confondus, ce nombre est passé de 468000 à 404000 . Les autorités de tutelle justifient ce mouvement par une nécessaire réorganisation de l'offre de soins sur le territoire. Il traduit aussi l'évolution structurelle des formes de prise en charge, qui se tournent de plus en plus vers des alternatives à l'hospitalisation à temps complet (diminution des long séjours, hospitalisation ambulatoire, etc.). Ce sont surtout les capacités d'accueil en long séjour qui ont subi une forte baisse, passant de 80000 lits en 2003 à 31000 lits en 2016. Cette baisse résulte de l'application de la circulaire de novembre 2008 relative à la partition des unités de soins de longue durée (USLD) afin de transférer les prises en charge de longue durée vers le secteur médico-social (Ephad) financées en partie par les collectivités territoriales.

Tableau 5 - Évolution du nombre de lits d'hospitalisation complète entre 2003 et 2016 (Source : adapté de Rapport Drees 2018)

\begin{tabular}{|c|c|c|c|c|c|c|c|c|c|c|c|c|c|c|c|}
\hline & & 2003 & 2004 & 2005 & 2006 & 2007 & 2008 & 2009 & 2010 & 2011 & 2012 & 2013 & 2014 & 2015 & 2016 \\
\hline \multirow{5}{*}{$\begin{array}{l}\text { Nombre de lits } \\
\text { d'hospitalisatio } \\
\text { n complete }\end{array}$} & $\mathrm{MCO}$ & 236407 & 233832 & 232163 & 229883 & 228664 & 225675 & 225350 & 224385 & 223324 & 222031 & 219968 & 216599 & 213510 & 210003 \\
\hline & Psychiatrie & 59590 & 59027 & 58585 & 58004 & 57640 & 57362 & 57131 & 57248 & 56950 & 57622 & 58001 & 57863 & 57503 & 57335 \\
\hline & SSR & 92432 & 92489 & 92028 & 94621 & 96451 & 98152 & 99227 & 100506 & 101226 & 102635 & 103434 & 104820 & 105593 & 105514 \\
\hline & USLD & 79989 & 76795 & 72399 & 68602 & 67860 & 61819 & 47966 & 34571 & 32704 & 32552 & 31803 & 31639 & 31639 & 31396 \\
\hline & Ensen & 468418 & 462143 & 455175 & 451110 & 450615 & 443008 & 429674 & 416710 & 414204 & 414840 & 413206 & 410921 & 408245 & 404248 \\
\hline
\end{tabular}

MCO : médecine, chirurgie, obstétrique et odontologie ; SSR : soins de suite et de réadaptation ; USLD : unité de soins de longue durée. Champ > France métropolitaine et DROM (y compris Saint-Martin, Saint-Barthélemy et Mayotte à partir de 2011)

En parallèle, depuis 15 ans, l'hospitalisation partielle a augmenté de plus 50\% (tableau 6). Un nombre croissant de procédures médicales sont désormais effectuées en dehors du cadre traditionnel de l'hospitalisation à temps complet. Ceci est expliqué par le développement 
d'innovations médicales et technologiques qui permettent des prises en charge ambulatoires ou à la journée (interventions chirurgicales, explorations endoscopiques, chimiothérapie ou radiothérapie, etc.). Les modifications des tarifications hospitalières ne sont pas analysées comme des déterminants de cette situation pourtant celles-ci intervenant annuellement, notamment pour l'ambulatoire, obligent les établissements à privilégier ce type de suivi en transférant une partie de la prise en charge hospitalière à la médecine de ville (généralistes, infirmières, soins à domicile, etc.) ou au secteur médico-social (Ephad, Hébergement longue durée, etc.). L'offre de soins territoriale pourrait bénéficier de ce transfert d'activité entre établissements de santé et secteur médical et médico-social si l'accessibilité à ces services de proximité n'était pas, de son côté, limitée par les objectifs de l'ONDAM ${ }^{17}$, générant, de fait, un recours accru aux services d'urgence.

Tableau 6. Évolution du nombre de places d'hospitalisation partielle entre 2003 et 2016 (Source : adapté de rapport Drees 2018)

\begin{tabular}{|c|c|c|c|c|c|c|c|c|c|c|c|c|c|c|c|}
\hline & & 2003 & 2004 & 2005 & 2006 & 2007 & 2008 & 2009 & 2010 & 2011 & 2012 & 2013 & 2014 & 2015 & 2016 \\
\hline \multirow{4}{*}{$\begin{array}{l}\text { Nombre de places } \\
\text { d'hospitalisation } \\
\text { partielle }\end{array}$} & MCO & 17964 & 18803 & 19577 & 20527 & 21763 & 23490 & 24942 & 26594 & 27922 & 29690 & 32264 & 31988 & 31448 & 32469 \\
\hline & Psychiatrie & 26382 & 26612 & 26552 & 26741 & 27156 & 27725 & 27995 & 28285 & 28424 & 28871 & 29065 & 29245 & 29357 & 29657 \\
\hline & SSR & 5017 & 5203 & 5664 & 6028 & 6983 & 7510 & 7818 & 8236 & 8797 & 9488 & 10613 & 11303 & 11984 & 12520 \\
\hline & Ensemble & 49363 & 50618 & 51793 & 53296 & 55902 & 58725 & 60755 & 63115 & 65143 & 68049 & 71942 & 72536 & 72789 & 74646 \\
\hline
\end{tabular}

\section{Les urgences : un exemple du lien dissymétrique entre territorialisation et financement des établissements de santé}

Depuis la réforme du financement hospitalier de 2004, le système de financement des urgences est mixte, avec:

- un forfait annuel à chaque service d'urgences autorisé (prévu pour un nombre de passage de 12500 par an), permettant de couvrir ses charges minimales de fonctionnement.

- un tarif par passage, dès lors que celui-ci n'est pas suivi d'une hospitalisation MCO dans l'établissement.

En 2016, en France métropolitaine et dans les DROM, il existe 719 structure urgences alors qu'il y en avait 750 en 2011. Cela dénote une relative stabilité dans la territorialisation des services d'urgence qui permet une bonne couverture du territoire mais avec des disparités importantes entre les régions. Selon la Drees, près de 4 millions de personnes, soit $6 \%$ de la population, résidaient encore à plus de 30 minutes d'un service d'urgences ou d'un SMUR fin 2015 (Drees 2017). Un million de personnes se trouvaient également à plus de 30 minutes d'un hélicoptère d'urgences (ibid.).

La faiblesse de l'activité des services d'urgence en zones rurales, compte tenu du mode financement de ces services, entraîne la suppression mécanique de crédits. Depuis la loi HPST (2009), le financement des structures d'urgences se fait en effet par site et non au niveau global de l'établissement ou du GHT, ce qui interdit toute mutualisation et pénalise les petites structures.

Les zones rurales se trouvent d'autant plus pénalisées que l'un des principaux acteurs de la permanence des soins non hospitaliers, SOS Médecins, ne fonctionne qu'en zone urbaine et péri-urbaine. Le modèle de fonctionnement de l'association nécessite en effet une certaine densité de population (60 000 habitants) afin de générer un nombre suffisant d'actes ${ }^{18}$..

Mais plus encore, la création l'ONDAM, qui encadre les dépenses de santé de ville (médecine générale, infirmières, kinésithérapeutes), a créé les conditions d'une limitation progressive de 
l'intervention de ces professions dans le traitement des urgences. Ceci explique en grande partie La progression continue des passages aux urgences depuis 20 ans (graphique 1).

\section{Graphique 1. Nombre de passage aux urgences entre 1996 et 2016 (Source : adapté de Drees 2018)}

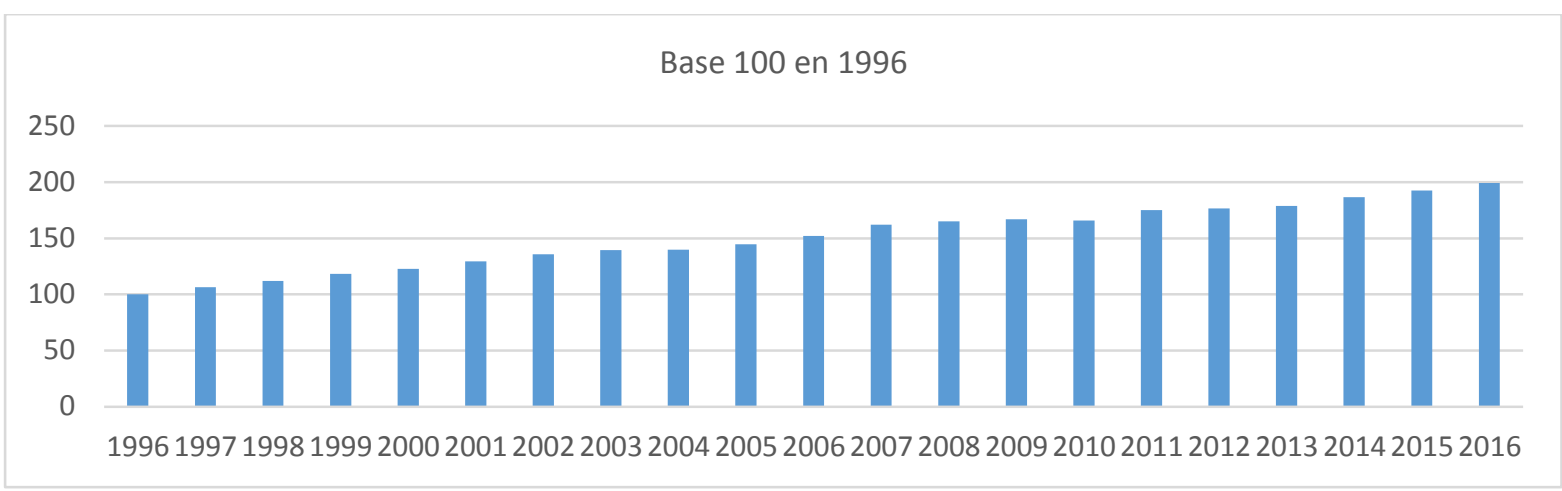

\section{Conclusion}

Nos analyses montrent des évolutions dissymétriques entre les modes de financement et la territorialisation des établissements de santé. D'un côté, il apparait que, depuis 20 ans, les modes de financement des établissements de santé ont permis une augmentation de l'activité de ces établissements permettant d'accueillir plus de patients et d'augmenter leur nombre de passages dans les hôpitaux et cliniques. Ceci peut être perçue, à la fois, comme une amélioration de la prise en charge des patients et comme une mesure de santé publique signifiant que les usagers pourraient être mieux, et plus efficacement, soignés dans leurs territoires. Cependant, d'un autre côté, ce constat peut aussi laisser transparaitre une recrudescence des inégalités d'accès à cette offre de soin. De nombreuses études ont montré que la territorialisation de la santé représentait un facteur de creusement ou de réduction de ces inégalités dépendant de déterminants liés aux patients et à leurs environnements (Fleuret, 2003 ; Amat-Roze, op.cit.). A côté de ces déterminants «externes » de la territorialisation de la santé, nous avons voulu vérifier dans quelles mesures les modalités de financement des établissements de santé pouvait constituer un déterminant plus «interne » au système de santé. Par une étude exhaustive de l'évolution de l'offre de soins et de la situation financière de ces établissements, nous avons révélé des éléments qui laissent transparaitre que les évolutions intenses qu'ont connues les modes de financement des établissements au cours des 15 dernières ont influencé de manière structurelle la capacité d'adaptation de l'offre de soins territoriale à sa patientèle. Nous suggérons ainsi qu'au moins deux éléments de financement ont produit des changements majeurs dans la territorialisation des établissements de santé : le suivi et le contrôle de l'activité des établissements, d'une part, la gestion par objectifs des dépenses de santé (ONDAM) et des budgets des établissements (CPOM, EPRD, PPI), d'autre part.

Ces résultats prennent la forme d'hypothèses de travail pour des études futures. Un prolongement naturel de ce travail devrait consister, s'il y a lieu, par une étude plus quantitative, à mesurer le niveau de corrélation ou de complémentarité entre les évolutions décrites dans cette étude.

\section{Bibliographie}


Amat-Roze, J. M. (2011). «La territorialisation de la santé: quand le territoire fait débat ». Hérodote, (4), 13-32.

Barlet M., Coldefy M., Collin C., Lucas-Gabrielli V. (2012). « L'Accessibilité potentielle localisée (APL) : une nouvelle mesure de l'accessibilité aux soins appliquée aux médecins généralistes libéraux en France », Irdes, Document de travail n ${ }^{\circ} 51$

Com-Ruelle L., Faure H., Tonnellier F. (1989). L'attraction de l'Assistance Publique Hôpitaux de Paris : L'hospitalisation de court séjour - Evolution 1981-1987. Rapport Credes, $\mathrm{n}^{\circ} 339$, décembre.

Comber, A.J., Brunsdon C., Radburn R. (2011). “A Spatial Analysis of Variations in Health Access: Linking Geography, Socio-economic Status and Access Perceptions". International journal of health geographics, 10:44.

Chauveau, S. (2011). "Quelle histoire de l'hôpital aux $\mathrm{XX}^{\mathrm{e}}$ et $\mathrm{XXI}^{\mathrm{e}}$ siècles ? ». Les Tribunes de la santé, 33,(4), 81-89. doi:10.3917/seve.033.0081.

De Pouvourville, G. (1990). «La régulation financière de l'hôpital par les DRG: enjeux, principes, faisabilité ». Sciences sociales et santé, 8(2), 33-65.

Drees - Insee (2014) « Réforme du financement des hopitaux publics : quels impacts sur leur niveau d'activité » études et résultats, ${ }^{\circ} 862$, janvier

Drees (2018) «Les établissements de santé », Panorama de la santé - édition 2018

Drees (2017) «Les établissements de santé », Panorama de la santé - édition 2017

Fleuret, S. (2003). «Recomposition du paysage sanitaire: quand les exigences territoriales rejoignent les enjeux de santé ». Géocarrefour, 78(3), 239-245.

Fleuret, S. (2015). «Construction locale de la santé: quels sont les facteurs de réussite des projets locaux de santé communautaire? » L'Espace Politique. Revue en ligne de géographie politique et de géopolitique, (26).

Haschar-Noé, N., Salaméro, É. (2016). «La fabrication d'un contrat local de santé «expérimental». Négociations et compromis sous tensions ». Sciences sociales et santé, 34(2), 81-105.

Irdes (2012) Le territoire, un outil d'organisation des soins et des politiques de santé ? Évolution de 2003 à 2012, Sous la direction de M. Coldefy etV. Lucas-Gabrielli , n 175

Irdes (2016) Pratiques spatiales d'accès aux soins. Rapport de l'IRDES, n565 sous la direction de Lucas-Gabrielli, V., Pierre, A., Com-Ruelle, L., \& Coldefy, M.

Lacoste O., Spinosi L. (2002). Distance, proximité, accessibilité, attraction et recours de la population, ORS NPDC

Lefebvre H., (1974). La production de l'espace, Paris, Éditions Anthropos.

McGuirk MA., Porell F.W. (1984). "Spatial Patterns of Hospitals Utilisation: The Impact of Distance and Time”. Inquiry, 21, pp 84-95 (Spring). 
Mériade, L., Nautre, B., Rochette C., Talbot D. (2017) "Les effets de la Proximité sur l'exécution des politiques publiques : L'exemple d'un Centre de Lutte contre le Cancer" politiques et Management public, 34/1 janvier -juin 2017, p. 143-162

Moschetti K. (2005). Quelle place pour les préférences des patients dans la régulation du système hospitalier ? Thèse pour le doctorat d'état en économie, Université de la Méditerranée.

Pilkington H. (2012). "Choice in Maternity Care: Associations with Unit Supply, Geographic Accessibility and User Characteristics". International Journal of Health Geographics, 11:35.

Power E., (2005), "Determinants of healthy eating among low-income Canadians", Canadian journal of public health, vol. 96, $13 \mathrm{p}$.

Rican, S., Vaillant, Z. (2009). «Territoires et santé: enjeux sanitaires de la territorialisation et enjeux territoriaux des politiques de santé ». Sciences sociales et santé, 27(1), 33-42.

Salze et al. (2011). "Estimating Spatial Accessibility to Facilities on the Regional Scale: An Extended Commuting-based Interaction Potential Model". International Journal of Health Geographics, 10:2 doi:10.1186/1476-072X-10-2.

Vallée J., Le Roux G., Chaix B., Kestens Y., Chauvin P. (2014). "The 'Constant Size Neighbourhood Trap' in Accessibility and Health Studies”, Urban Studies, I-20.

Victoor A., Delnoij D. MJ, Friele R., Rademakers J. (2012). "Determinants of Patient Choice of Healthcare Providers: A Scoping Review”. BMC Health Services Research, 12:272.

Wellstood W., Wilson K., Eyles J. (2006). "Reasonnable Access to Primary Care: Assessing the Role of Individual and System Characteristics". Health and Place, 12: 121-130.

Zeitlin J., Combier E., Levaillant M., Lasbeur L., Pilkington H., Charreire H., Rivera L. (2011) "Neighbourhood Socio-economic Characteristics and the Risk of Preterm Birth for Migrant and Non-migrant Women: A Study in a French District". Paediatr Perinat Epidemiol, 25(4):347-356.

\footnotetext{
${ }^{1}$ «Hôpital, patient, santé, territoire » (2009)

${ }^{2}$ Agences Régionales d'Hospitalisation (1997) remplacées par les Agences régionales de Santé (2010)

${ }^{3}$ Schéma Régional d'Organisation des Soins (2009)

${ }^{4}$ Groupements Hospitaliers de Territoires

${ }^{5}$ L'article L6111-1 du Code de la santé publique définit les établissements de santé comme des structures publiques $(62 \%$ des lits) ou privés à but lucratif (24\% des lits) et non lucratif (14\%) qui participent à la mise en œuvre de la politique de santé publique à travers leurs activités médicales et d'hospitalisation.

${ }^{6}$ Direction de la recherche, des études, de l'évaluation et des statistiques du Ministère de la Santé

${ }^{7}$ Institut de recherche et de documentation en économie de la santé

${ }^{8}$ Institut national de la statistique et des études économiques

${ }^{9}$ Direction Générale des Finances Publiques

${ }^{10}$ Hôpital, Patients, Santé, Territoire

${ }^{11}$ dans un premier temps pour des maladies chroniques telles que le diabète et l'insuffisance rénale

${ }^{12}$ Mission d'Intérêt Général et Aide à la Contractualisation

${ }^{13}$ La DREES et l'INSEE ont développé une méthodologie d'analyse de l'accessibilité des services de santé en ville ou à l'hôpital, qui mobilise principalement deux bases de données : le Programme de médicalisation des systèmes d'information (PMSI) pour les soins hospitaliers et le Système national d'informations inter-régimes de l'Assurance maladie (SNIIR-AM) pour les soins de ville.

${ }^{14}$ Contrat Pluriannuel d'Objectifs et de Moyens

${ }^{15}$ Etat Prévisionnel des Dépenses et des Recettes construit annuellement

${ }^{16}$ Projet Pluriannuel d'Investissement
} 
${ }^{17}$ Objectif National des Dépenses d'Assurance Maladie (1996)

18 «Les urgences hospitalières, miroir des dysfonctionnements de notre système de santé », rapport d'information du sénat $n^{\circ}$ 685, 26 juillet 2017 